\section{Laryngeal Adenoid Cystic}

\section{Carcinoma: A rare neoplasm}

\section{with still undefined treatment}

\section{aspects. Case report and literature review}

\author{
Gianluigi Mariano Grilli', Rossana Giancaspro', Valeria \\ Fiore ${ }^{1}$, Anna Del Colle ${ }^{2 *}$, Luciano Enrico Renato Magaldi ${ }^{1}$ \\ and Giuseppe Pannone ${ }^{3}$
}

'Department of Otolaryngology, University of Foggia, Foggia, Italy

${ }^{2}$ Department of Respiratory Disease, University of Foggia, Foggia, Italy

${ }^{3}$ Department of Anatomic Pathology and Cytopathology, University of Foggia, Foggia, Italy
Received: 03 July, 2020

Accepted: 10 July, 2020

Published: 11 July, 2020

*Corresponding author: Anna Del Colle, MD, Department of Respiratory Disease, University of Foggia, Viale degli Aviatori, 2, 71122, Foggia, Italy, Tel: +393405744845; E-mail: annadelcolle@gmail.com Keywords: Adenoid cystic carcinoma; ACC; Larynx; Laryngeal; Salivary gland malignancies https://www.peertechz.com

\section{Check for updates}

\begin{abstract}
Adenoid Cystic Carcinoma (ACC) is a rare malignant tumor, accounting for about $1 \%$ of head and neck malignancies and $10 \%$ of salivary gland cancers. The laryngeal localization of ACC is particularly rare because of the paucity of accessory salivary glands in this anatomical district. Prevalence is highest in the fifth and sixth decade of life with a slight female predilection and aetiology is unknown. Since laryngeal ACC characteristically grows slowly and occurs in the subglottis, the diagnosis, which is based on the combination of physical examination with fiberoscopy and neck-chest Computerized Tomography (CT), is often delayed. Nowadays the debate is still open about which is the best comprehensive therapy of this tumor. We present the case of a laryngeal ACC in a 68 year-old-woman, treated with total laryngectomy together with bilateral neck dissection and a literature review made with the purpose to point out the most recent advancements towards the definition of a gold-standard therapy. Laryngectomy results as the first choice treatment, while chemotherapy has minimal effects on this neoplasm and radiotherapy alone does not show the capacity to lead to complete resolution, but there is still the need of further studies to clarify the usefulness and the role played by neck dissection and postoperative radiotherapy.
\end{abstract}

\section{Introduction}

Adenoid Cystic Carcinoma (ACC) is a rare malignant tumor, accounting for about $1 \%$ of head and neck malignancies and $10 \%$ of salivary gland cancers [1]. ACC most commonly affects the major salivary glands and the minor salivary glands of the oral and sinonasal cavities. Laryngeal ACC, which arises from the submucosal glandular elements of the larynx, is extremely rare because of the paucity of accessory salivary glands in this anatomical district [2]. The laryngeal location reflects the density of distribution of the subepithelial glands, which is higher in the supraglottic and subglottic areas: $64 \%$ of these tumors occur in the subglottis, $25 \%$ in the supraglottis, $5 \%$ in the glottis, and $6 \%$ in the transglottic area [3]. Symptoms depend on the location of the tumor: supraglottic tumors usually present with dysphagia and hoarseness, subglottic tumors with stridor and dyspnea [4]. Since ACCs characteristically grow slowly with perineural extension, advanced cases could be associated with neck pain [5]. ACC has a predisposition to local recurrence and distant metastases, particularly affecting the lungs. The aetiology of ACC remains unknown. Prevalence is highest in the fifth and sixth decade of life with a slight female predilection [6]. The diagnosis of laryngeal ACC is based on the combination of physical examination with fiberoscopy and neck-chest Computerized Tomography (CT). Nowadays the treatment of this tumor is challenging. Surgery is the first choice treatment, eventually combined with postoperative radiotherapy treatment in the case of risk factors for recurrence 
(positive surgical margins, vascular embolization or perineural invasion) [6].

We present the rare case of a laryngeal ACC in a 68 yearold-woman, treated with total laryngectomy together with bilateral neck dissection and a literature review.

\section{Case report}

A 68-year-old Caucasian woman was admitted to ENT Department of University Hospital "Ospedali Riuniti" of Foggia with complaint of progressive dysphonia and dyspnea since the last 5 months. The patient was a heavy smoker (20 cigarettes/day for 40 years) and had previously been diagnosed with papillary thyroid carcinoma, which had been treated with partial thyroidectomy and right Selective Neck Dissection (SND) followed by radiometabolic therapy. She had no history of cough, prelaryngeal pain and dysphagia. Fiberoptic laryngoscopy detected a bulging mass arising from the subglottic area involving the right true vocal cord, with lack of mobility of the right larynx and laryngeal stenosis of $80 \%$. Laryngeal mucosa was intact. On physical examination, the patient showed inspiratory laryngeal stridor. She had no palpable neck mass or lymphadenopathy. Clinical staging was T3NoMo (stage III, according to the Union for International Cancer Control/American Joint Committee on Cancer staging system). CT of the neck with contrast medium revealed the presence of a solid lesion in the right subglottic area caudally extended up to the first tracheal ring and cranially up to the ipsilateral vocal cord (Figure 1). In addition, CT of the chest revealed the presence of three small pulmonary nodules of a suspicious nature. In order to identify any pulmonary metastases, the patient underwent a total-body positron emission tomography-computed tomography (PET-CT) with contrast medium, which confirmed the presence of a solid lesion in the right subglottic area with hyperaccumulation of the tracer (standardized uptake volume, 8) and two areas of tracer uptake in the right submandibular region, inside residual level II area (standardized uptake volume, 3,2). PET-CT did not showed any pulmonary tracer uptake. The findings on chest $\mathrm{X}$ - ray were normal. We performed a lesion biopsy that was consistent with an ACC of the larynx (tumor cells were positive for CD117, P63 and smooth muscle actin). Subsequently, the patient underwent total laryngectomy associated with SND (residual level II, right side; levels II-III-IV, left side). The surgical margins resulted free of tumor. The final pathology report showed negative margins. Specimen showed intraluminar (sub-mucosal) growth with involvement of thyroid cartilage (Figure 2) and perineural invasion (Figure 3) involving the terminal branches of right laryngeal nerves, which are removed together with the larynx in a total laryngectomy. All dissected neck lymph nodes were free of metastases. Thus, the tumor was pathologically staged as T4aNoMo (stage IVa). Ten days post-operatively, the patient was discharged. The patient refused adjuvant radiotherapy. Our follow-up was performed monthly for the first six months and every 2 months for the next half of the first year, then every four months. Every session included an endoscopic evaluation of the pharynx, upper esophagus and tracheal space through the tracheostomy, together with clinical examination of oral cavity and neck. We also planned two neck and thorax CT scans with contrast medium to perform 6 and 18 months after surgery,
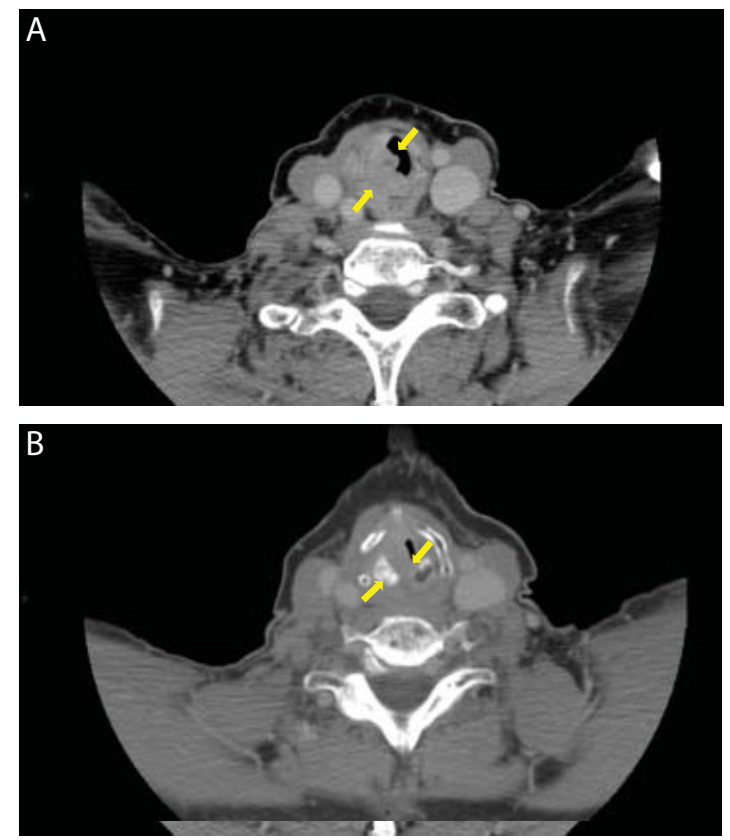

Figure 1A,B: Neck CT study without contrast medium, in cross section, shows an extensive and irregular solide lesion of the subglottic region (A - yellow arrows) that, with a maximum length of almost $3 \mathrm{~cm}$, cranially incorporates the ipsilateral vocal cord ( $B$ - blue arrow). Homolaterally, the image shows a thickening of the paralaryngeal planes (red arrow) and sclerosis of the right arytenoid cartilage (green arrow).

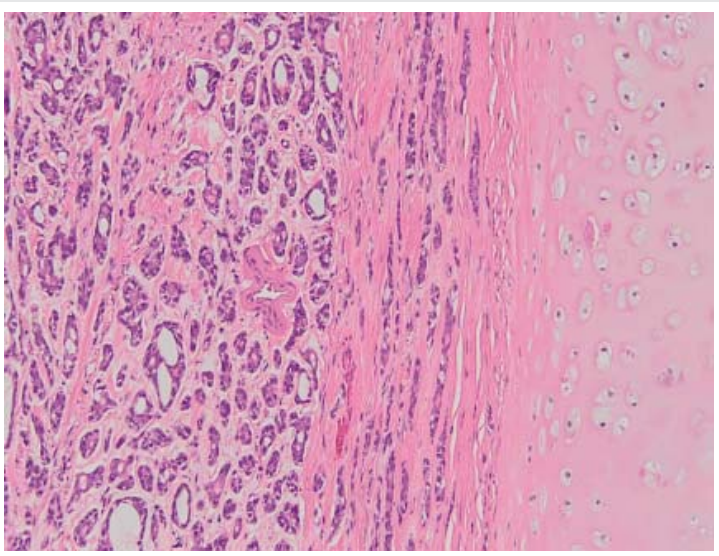

Figure 2: Hematoxylin-eosin overview showing the predominantly cribriform pattern and the infiltration of the perichondrium of the thyroid cartilage (Hematoxylin-Eosin $4 \mathrm{X})$

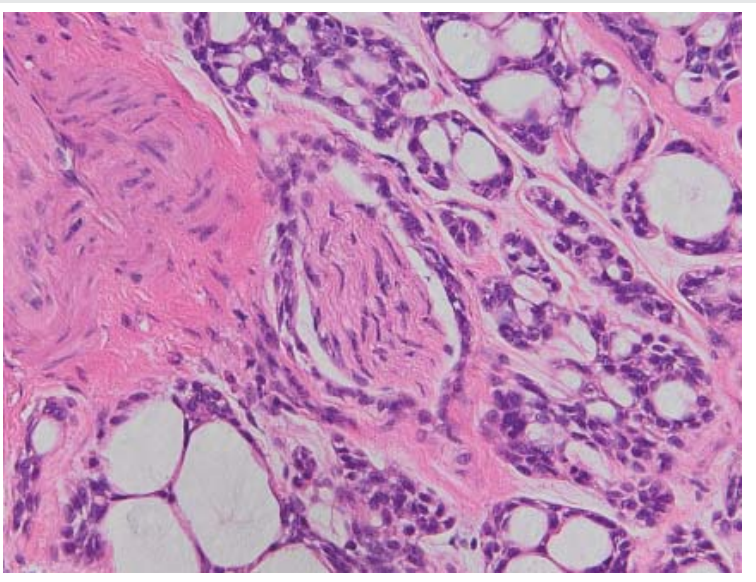

Figure 3: Higher magnification image showing the microscopic aspects of perineural invasion (Hematoxylin-Eosin 20X) 
in order to monitor the evolution of pulmonary nodules: both CTs showed no modifications. After two years of postoperative follow-up no clinical, endoscopic and radiological evidence of local recurrence and distant metastases was detected.

\section{Discussion}

Adenoid cystic carcinoma is a malignant salivary gland tumor that originates from salivary glands or mucinous glands of the upper aerodigestive tract. Laryngeal ACC is very rare because of the relative lack of accessory salivary glands in the mucosa of laryngeal-tracheal tract. We made a literature review that revealed about 96 well-documented cases of laryngeal ACC. In particular, Ricciardiello and his colleagues reported only 81 cases between 2001 and 2015 [4]. After that year we found other 16 cases, making a PubMed research using the key words 'adenoid cystic carcinoma' together with 'larynx' and 'laryngeal'. The features of these cases are summarized in Table 1. The most common primary site in larynx is, as in our case, the subglottic region. It usually occurs in female patients aged between 50 and 60 years old. Etiology is already unknown. Interestingly, unlike squamous cell carcinoma (SCC), which is the most common laryngeal malignancy, smoking does not predispose patients to ACC. Therefore, the reduction over the years in the incidence of other laryngeal malignancies, despite the constant incidence of ACC, could be related to the decreased popularity of smoking [7]. ACC has histological features similar to those of the basaloid SCC, a recognized variant of SCC. Even the symptoms of SCC do not differ greatly from those of ACC Thus, a preoperative histopathological analysis is essential in order to exclude other differential diagnosis such as basaloid SCC, which has a worse prognosis. Furthermore, ACC may be divided into three histologic patterns: tubular, cribriform and solid. The most common is the cribriform pattern $(46,3 \%)$, followed by solid $(38,3 \%)$ and tubular $(14,9 \%)$ patterns [8]. In our case, the neoplasm was diagnosed in a 68-year- old female heavy smoker and exhibited a classic cribriform growth pattern. Since ACC usually occurs as a large asymptomatic and non-ulcerated submucosal mass, the diagnosis is often delayed. Indeed, early ACC could be clinical misdiagnosed as asthma and other chronic tracheobronchial diseases or laryngeal amyloidosis [9]. When our patient was referred to our ENT Department the disease was already at an advanced stage, despite dyspnea and dysphagia appeared a few months before. She was initially diagnosed with clinical stage III (T3NoMo). We made an accurate preoperative staging with fiberoptic laryngoscopy and CT, a very useful imaging procedure that can assess the primary tumor location, extra-luminal extension and metastases. In our case, CT with contrast medium showed the presence of a solid lesion in the right subglottic area caudally extended up to the first tracheal ring and cranially up to the ipsilateral vocal cord. Furthermore, CT of the chest revealed the presence of three small $(<1 \mathrm{~cm})$ pulmonary nodules of a suspicious nature. Thus, we searched for lung metastatic lesions using PET-CT. In fact, local recurrences and distant metastases are common, especially in the lungs, liver, bone, lymph nodes and bones. However, the presence of lung metastases at the time of presentation should not prevent laryngectomy because ACC shows a very slow growth pattern and the patients can survive for a long time with the disease [10]. PET-CT with contrast medium confirmed the presence of a solid lesion in the right subglottic area and two areas of tracer uptake in the right submandibular region, but no pulmonary tracer uptake was shown. Our patient first underwent direct microlaringoscopy biopsy that confirmed laryngeal ACC and then, taking into account the age of the patient, the stage of local and loco-regional tumor expansion and the possibility of occult nodal metastases, we decided that total laryngectomy associated with SND would be the optimal therapeutic. Tumor staging was postoperatively classified as T4aNoMo.

Although our research documents a progressive increase in the number of laryngeal ACC reported in the literature during the last years as shown in Table 1 the treatment options appear to be still controversial.

There is a general consensus on surgery as the mainstay treatment modality. In particular, total laryngectomy is the recommended therapeutic procedure for this lesion, while partial laryngectomy has to be taken into account only for selected few patients with small and well- defined tumours6. On the contrary, we still found an ambiguous guidance about complementing or not the laryngectomy with neck dissection: Coca-Pelaz, et al. in their review consider that the incidence of occult neck metastases among patients with ACC is low (17\%) and report no survival advantage for patients who underwent Elective Neck Dissection (END) compared with those who did not [11], while Chang, et al. underline in their study the association between lymph node metastases and poor prognosis, considering elective neck dissection an adequate treatment for $\mathrm{T} 3$ and $\mathrm{T} 4$ cases [8]. In our case, evaluated the clinical staging, in absence of particular comorbidities, we decided to remove the residual II level on the right side in order to analyze the two areas of PET tracer uptake in the right submandibular region and perform END on the remaining side.

ACC are radiosensitive but usually not radiocurable neoplasms. For this reason radiotherapy alone is the main therapeutic option only for tumors that cannot be resected, while the role of adjuvant radiotherapy is still debated. It seems to improve prognosis in case of lymph node metastasis, high tumor grade, positive surgical margin and advanced tumor stage $[8,12]$. Our patient refused adjuvant radiotherapy, therefore we excluded this option from our treatment planning.

Chemoterapy applications in laryngeal ACC appear to be more clearly defined. It has minimal effects on these tumors, playing the role of a suitable therapeutic alternative only for advanced tumors with distant metastases [4]. Consequently, our patient was not subjected to chemotherapy.

\section{Conclusion}

Although ACC constitutes a minority of laryngeal malignancies, it must be suspected in cases of laryngeal masses, especially in relatively young females. Most patients are diagnosed at an advanced stage. CT scan with contrast medium is useful to assess tumor extent and growth patterns and a preoperative histopathological analysis is essential in order to confirm the diagnosis. Being a rare finding in the laryngeal district, therapeutic options are controversial. We believe that surgical resection with wide safe margin is the optimal therapeutic, eventually complemented with radiotherapy, while neck dissection is an option to evaluate 
Table 1: Previous well-documented cases of laryngeal ACC, between 2015 and 2020.

\begin{tabular}{|c|c|c|c|c|c|}
\hline First author & Year & Case(s), n & $\begin{array}{l}\text { Primary tumor } \\
\text { localization }\end{array}$ & Recurrence sites (local-regional/metastases) & Survival \\
\hline Cui, et al. [13] & 2019 & 3 & Subglottis & $\begin{array}{l}\text { Distant metastases ( } 1 \text {, pulmonary metastases } \\
\text { after } 6 \text { years), none ( } 2 \text { ) }\end{array}$ & $\begin{array}{l}2 \text { patients alive (2 NED- } 2 \text { years follow-up), } 1 \\
\text { patient succumbed }\end{array}$ \\
\hline Naim, et al. [14] & 2019 & 1 & Subglottis & ND & ND \\
\hline Wang, et al. [3] & 2018 & 1 & Subglottis & None & NED- 17 months follow-up \\
\hline Ricciardiello, et al. [4] & 2018 & 1 & Subglottis & None & NED- 2 years follow-up \\
\hline Shirian, et al. [15] & 2017 & 1 & Larynx & ND & ND \\
\hline Kashiwagi, et al. [9] & 2016 & 1 & Subglottis & None & NED- 2 years follow-up \\
\hline Li, et al. [1] & 2015 & 2 & Subglottis & $\begin{array}{c}\text { Distant metastases ( } 1 \text {, pulmonary and bone } \\
\text { metastases after } 15 \text { months), regional } \\
\text { recurrence( } 1)\end{array}$ & 2 patient succumbed \\
\hline Liu, et al. [16] & 2015 & 6 & $\begin{array}{l}\text { Supraglottis(2), } \\
\text { subglottis (4) }\end{array}$ & $\begin{array}{c}\text { Distant metastases ( } 1 \text {, pulmonary metastases, } 1 \\
\text { liver metastases), none (4) }\end{array}$ & $\begin{array}{c}5 \text { patients alive ( } 4 \text { NED- Follow-up period 1-6 } \\
\text { years), } 1 \text { patient succumbed }\end{array}$ \\
\hline
\end{tabular}

taking into account general and clinic features of the specific patient. Close and long-term follow-up is mandatory to rule out tumor recurrence.

\section{Statement of ethics}

This research was conducted ethically in accordance with the World Medical Association Declaration of Helsinki. The subject has given her written informed consent to publish her case (including publication of images).

\section{Author contributions}

Gianluigi Mariano Grilli: writing and review of the manuscript Rossana Giancaspro : writing of the manuscript.

Valeria Fiore: bibliography analysis.

Anna Del Colle: writing of the manuscript.

Luciano Magaldi: bibliography analysis.

Giuseppe Pannone: images development, review of the manuscript.

\section{References}

1. Li G, Chen J, Zhang S, Lin J, Kong F, et al. (2015) Adenoid cystic carcinoma of the larynx: A report of two cases. Oncol Lett 10: 2303-2306. Link: https://bit.ly/3ebFtT7

2. Xiao R, Sethi RK, Feng AL, Fontanarosa JB, Deschler DG (2019) The role of elective neck dissection in patients with adenoid cystic carcinoma of the head and neck. Laryngoscope 129: 2094-2104. Link: https://bit.ly/2ZibXqS

3. Wang Q, Yu XS, Liu Y, Chen H (2018) Adenoid cystic carcinoma in ventricle of larynx: An interesting case. Med (United States) 97: e13622. Link: https://bit.ly/2BWhOnR

4. Ricciardiello F, Addeo R, Di Lullo AM, Abate T, Mazzone S, et al. (2018) Adenoid cystic carcinoma of the larynx in a 70-year-old patient: A case report. Oncol Lett 16: 2783-2788. Link: https://bit.ly/2CpNrLu
5. Kolahdouzan M, Abtahi SHR, Abedini L, Jafari HR, Mostafapour E (2017) The case report of laryngeal adenoid cystic carcinoma: A rare entity. Int J Cancer Manag 10. Link: https://bit.ly/2W4C8il

6. Zvrko E, Golubović M (2009) Laryngeal adenoid cystic carcinoma. Acta Otorhinolaryngol Ital 29: 279-282. Link: https://bit.ly/2CjWw8o

7. Dubal PM, Svider PF, Folbe AJ, Lin HS, et al. (2015) Laryngeal adenoid cystic carcinoma: A population-based perspective. Laryngoscope 125: 2485-2490. Link: https://bit.ly/3egHgGG

8. Chang CF, Hsieh MY, Chen MK, Chou MC (2018) Adenoid cystic carcinoma of head and neck: A retrospective clinical analysis of a single institution. Auris Nasus Larynx 45: 831-837. Link: https://bit.ly/3ei4Na4

9. Kashiwagi T, Kanaya H, Konno W, Goto K, Hirabayashi H, et al. (2016) Adenoid cystic carcinoma of the larynx presenting with unusual subglottic mass: Case report. Auris Nasus Larynx 43: 562-565. Link: https://bit.ly/321VPLF

10. Moukarbel RV, Goldstein DP, Sullivan BO, Gullane PJ, Brown DH, et al. (2008) Adenoid cystic carcinoma of the larynx: a 40-year experience. Head Neck 30: 919-924. Link: https://bit.ly/2DuwPmf

11. Coca-Pelaz A, Barnes L, Rinaldo A, Cardesa A, Shah JP, et al. (2016) Cervical Lymph Node Metastasis in Adenoid Cystic Carcinoma of the Larynx: A Collective International Review. Adv Ther 33: 553-579. Link: https://bit.ly/3fhysS7

12. Hsu AAL, Tan EH, Takano AMP (2015) Lower Respiratory Tract Adenoid Cystic Carcinoma: Its Management in the Past Decades. Clin Oncol 27: 732-740. Link: https://bit.ly/2Dn5BxG

13. Cui Y, Bi L, Sun L, Wang X, Zhu Z (2019) Laryngeal adenoid cystic carcinoma: Three cases reports. Med (United States) 98: e18177. Link: https://bit.ly/2ZkgDwt

14. Naim A, Hajij A, Abbad F, Rami A, Essaadi M (2019) Rare location of head and neck adenoid cystic carcinoma. Pan Afr Med J 34: 33. Link: https://bit.ly/209cDZf

15. Shirian S, Maghbool M, Aledavood A, Negahban S, Khademi B, et al. (2017) Adenoid Cystic Carcinoma of the Larynx Presenting as a Thyroid Mass and Brief Literature Review. Acta Cytol 61: 237-241. Link: https://bit.ly/2Oci2yC

16. Liu W, Chen X (2015) Adenoid cystic carcinoma of the larynx: a report of six cases with review of the literature. Acta Otolaryngol 135: 489-493. Link: https://bit.ly/2W6Ftxz

Copyright: ๑ 2020 Grilli GM, et al. This is an open-access article distributed under the terms of the Creative Commons Attribution License, which permits unrestricted use, distribution, and reproduction in any medium, provided the original author and source are credited. 